\title{
Cosmic Intelligence and Black Holes
}

\author{
Vladimir A. Lefebvre \\ School of Social Sciences \\ University of California, Irvine \\ and \\ Yuri N. Efremov \\ Sternberg Astronomical Institute \\ Moscow State University, Moscow
}

\begin{abstract}
The paper is devoted to a new direction in SETI. After a general discussion of the field, the authors put forth the hypothesis that the black holes may serve as a physical substratum for intelligent beings. This hypothesis is based on four parallels between the brain-psyche system, on the one hand, and black holes, on the other. (1) The descriptions of brain and psyche, in the system brain-psyche, are complementary to each other, as descriptions by internal and external observers of a black hole in Susskind-t'Hooft's schema. (2) There is an aspect of the inner structure of a black hole in Kerr's model of the rotating black hole that is isomorphic to the structure of the human subjective domain in the psychological model of reflexion. (3) Both black holes and the brainpsyche system have a facet which can be represented using thermodynamic concepts. (4) The brain lends itself to a holographic description; as has been recently demonstrated by Susskind, black holes can also be described holographically. The authors speculate that the intelligent black holes can generate other black holes by triggering creation of massive stars from which new black holes arise after the stars' collapse. In addition, the authors analyze certain strange phenomena related to the birth of young massive stars which may or may not be connected with such a triggering.
\end{abstract}

\section{Introduction. The Search for Extraterrestrial Civilizations}

This article was written by two men no longer young. They first met a halfcentury ago in the school Astronomy Club of the Moscow Planetarium. Subsequently one of them became a professional astronomer, the other a psychologist and anthropologist. Like the many other young astronomers gathering every week in the Planetarium's Star Room, they were fascinated, among the other issues of this science, by the question of whether or not we are alone in the 
Universe. This question remains with us to this day, along with the memory of the stars' twinkling in the planetarium dome and the whole festive atmosphere of our first contact with knowledge. It was seemingly no accident that our fellow member N.S. Kardashev became one of the pioneers in the search for extraterrestrial civilizations.

Unfortunately, this problem has yet to acquire serious scientific status. One of the reasons for this is that the devotees of this field formulate their goals guided only by intuition and common sense. The proposal of hypotheses regarding the types of activity of extraterrestrial intelligence, in essence, is little different from the free inventions of science fiction writers. The search for cosmic civilizations will acquire the status of a strictly scientific undertaking only if it becomes possible to construct a theoretical model of the world, one of whose natural components would be the intelligent subject. Such a model should connect mind as a phenomenon with our physical understanding of the Universe, to shed light on the nature of the physical objects we seek to find.

Such an undertaking is fully suited to the spirit of the natural sciences at the turn of the new century. We are the witnesses of a new scientific revolution in physics comparable with the one whose results let to the appearance of quantum mechanics. The unification of quantum mechanics with the theory of relativity is taking place before our eyes. A storm of research is going on in two fields, whose code names are "quantum gravitation" and "string theory." In this article we attempt to conduct a series of theoretical and empirical arguments that this scientific revolution may culminate in something unexpected - the inclusion of the intelligent subject in a unified purview. And it is precisely such a unified model that may indicate to us the objective traits of intelligent subjects.

\section{The Silence of the Universe}

Today we possess a detailed view of the Universe, and nowhere do we find barriers to the spatial propagation of intelligent life, once it has appeared. Nonetheless, all attempts to identify extraterrestrial intelligence have so far been futile. Enrico Fermi's well-known question "Where are THEY?" turned the absence of any signs of alien intelligence into a scientific paradox that changed the very approach to this problem. It became clear that "the silence of the Universe" is a special phenomenon that must be explained. M. Hart and I.S. Shklovsky proposed a radical solution to the paradox: THEY are silent, because they simply are not there. Another radical solution lies in the reply - whoever was not silent, has ceased to exist - given by R. Zubrin at a conference in Colorado during a presentation of one of the authors of this paper. This deep thought had

been expressed before. Let us imagine, for example, that the higher forms of intelligence related to the lower forms as we do to harmful insects, like locusts. They are annihilated as soon as the radio picks them up.

The silence of the Universe, however, can also be explained in less radical terms, such as, for example, that the ages of comparable technological development of civilizations must coincide in order for them to recognize one another. 
The characteristic scale of technological development on Earth, whether you begin with Maxwell-Hertz or with Popov-Marconi, is only 100 or 150 years. Even starting with the ancient Greeks there has elapsed a period of time only one order greater, whereas the age of the oldest stars is greater by eight orders. We are unable to imagine the potential of humanity even 100 years from now, not to speak of a billion - that is, if the development of science, and of our own existence, will continue that long.

One way or another, it is hard to imagine another scenario than that intelligence first appeared in the course of biological evolution. Thus we may suppose that during some stage of technical development and comprehension comparable realities may appear in all civilizations throughout the Universe. The problem, it seems, lies precisely in the very rapid pace of technological development, and in the very great disparity, several billion years, in the ages of stars with an appropriate content of heavy elements, which entails a disparity in the age of planets on which life may appear, and so of the moments at which intelligent life appears. For this reason, the probability of finding two civilizations at the same time with comparable levels of development, permitting one of them to recognize the other, is very small, and the chances of finding intelligent siblings nearby are negligible.

(Many years ago one of the present authors shared with I.S. Shklovsky his observation, that we should pay attention to unusual radiation coming from opposite points in the heavens, in the hope that we may intercept precisely directed radio transmissions between stars. Yet one of the pioneers in the search for extraterrestrial civilizations became an extreme pessimist around this time, considering it most likely that we are alone in the Universe. His answer was a sad smile that requires, you see, that there be three civilizations on a single line...)

The tiny possibility of encountering intelligent siblings, however, may mean, that we have not noticed THEM simply because we've hardly looked. Further research is essential, along with further development of astronomy's tools for observation. A particularly promising avenue is the study of star clusters, whose stars have roughly the same age, and whose size is measured in light-weeks, such that the inhabitants of such clusters would be able to conduct practical exchanges of information. The power of their directed signals may be great enough, and we might be able to happen upon the extension of a radio beam. Globular clusters of about 15 billion years in age, containing millions of stars, would be the most interesting, if it weren't for two circumstances - their stars contain few heavy elements, and the density of their central parts is so great that the planets' orbits around stars may be unstable. In the search for life resembling ours, the best places to look are the old, dispersed clusters with a normal chemical abundance and ages less than 10 billion years, like M67 and NGC 188. 


\section{How Long Has Intelligence Existed?}

There are, however, reasons for remaining suspicious of all these possibilities. We cannot exclude the possibility that such notions as "life", "technology," and even "intelligence" are merely the reflections of weakly developed self-organizing cosmic systems. The higher levels of development are not subject to effective description using the conceptual tools of modern science. We may recall that B.N. Panovkin insisted on the possibility that different civilizations can have systems of signs and concepts of radically different types, such that mutual comprehension is fundamentally impossible.

Let us imagine that technologically and, consequently, scientifically developed civilizations like those on Earth are aware that in several centuries they will be transformed into objects of a wholly different kind, in relation to which expressions like "await a response from our intelligent siblings" have no meaning. In such a case the civilization will have no reason to send signals to anyone, or to seek to receive them, and this problem simply does not arise. Such a civilization is like a caterpillar which turns into a butterfly. The caterpillar, if it is aware that it will soon turn into a butterfly, will not send signals to other caterpillars. It is possible that the comprehension of such possibilities comes at the same time as the realization of technical means for the transmission of adequately long-distance signals. Thus we have the silence of the Universe.

The very notion of intelligence in relation to cosmic civilizations is often understood in too narrow a sense, as a system's ability to resolve difficult tasks which are essential for survival in a complex and sometimes hostile environment. In such a context the central question becomes one of whether intelligence is a characteristic only of biological systems. This problem was posed by A. Turing in the thirties and carefully considered by the founder of cybernetics, N. Wiener. In 1980, this theme was again sounded by F. Dyson in connection with his analysis of the problems of immortality, from the point of view of physics. Dyson used a functional definition of life in whose framework there is an essential structure of relations among elements, yet whose physical substratum is unimportant. He came to the conclusion that intelligent beings capable of changing the physical nature of their bodies may potentially exist eternally, but that in the distant future, when the entropy of the Universe becomes extremely high, they will have to interrupt their activity for every longer intervals, hibernating and waking up to the sound of a particular "alarm clocks." Nevertheless, in L. Kraus and G. Starkman (Scientific American, Nov. 1999) showed that the existence of such an "alarm clock" contradicts the principles of quantum mechanics. That means that each such intelligent system must eventually go to sleep forever ...

An intelligent system cannot exist eternally, but it can strive for a long life, on the cosmic scale of time. Our further analysis will touch upon the hypothesis about how highly-developed systems might resolve this problem. 


\section{The Invariants of Perfect Systems}

The most perfect systems that we know are ourselves. Therefore, let us take a look at our "self- description," as it has been developed in modern psychology. The human being is represented here in two ways. First of all, it is regarded from the external viewpoint as a particular object. This permits us to study human behavior and the human brain, in the hope of determing the objective links between them. The brain, in this aspect, appears as a complex information system. We must note that in the 1960's holographic means were proposed for the representation of this system (B. Jules and K. Pennington, P. Westpaik, K. Pribram). The peculiarity of psychological investigation, however, demands that we study the human being from a fundamentally different, internal viewpoint. We are interested not only in how the brain works, but also in how the human being feels this, what pictures appear before the eyes, as it evaluates and realizes itself. Psychiatry is wholly based not so much on models of the brain, as on sophisticated views of the inner subjective life. Throughout the 20th century numerous attempts have been made to cross the barrier separating the inner and outer viewpoints, to create a third, more general one. All these attempts have been unsuccessful in the end. We apparently must accept the idea, expressed by Nils Bohr, that the outer and inner views of a phenomenon of consciousness are in a complementary relationship.

A few years ago one of the authors of this article constructed a formal model of the subject in the act of choosing between two polar alternatives (V.A. Lefebvre, The Cosmic Subject. Moscow, Russian Academy of Sciences Institute of Psychology Press, 1997 (in English). Available at Amazon.). This model's predictions have since passed psychological tests (J. Adams-Webber, American Journal of Psychology, 1998, V.110, 527-541). The core of the model is a particular mathematical function, which can be deduced from several elementary propositions concerning the character of human activity. It turned out that this function possesses one remarkable characteristic. It can be represented as the repeated composition of a single simpler function, a representation, moreover, which is unique. This composition was naturally interpreted as a system of sequential images of the self, possessed by the subject. In other words, the subject possesses an image of itself, which, has, in turn, an image of itself, and so forth. Each image is a subject, which exists in the inner world of a certain observing subject. Therefore, the observer has two complementary points of view: one is external, and the other, is, from the standpoint of its image, internal. Moreover, it turns out that each image is a "mix" of two states - positive and negative.

As a theoretical analogy for the action of the subject this model employs a projection on some external "screen" of the subject's state. Such a projection can occur either as the creation of an external self-description, or as self-reproduction (the model does not distinguish between the two). The further analysis of this model permits us to reveal its formal connection with the functioning of neural networks, and also with the laws of thermodynamics. It turns out that if formal neurons in an active state are accidentally connected with other neurons, "infecting" them by their state, the dynamics of the system 
may be described by a differential equation whose solution is a function describing human reflexion (Lefebvre, 1998). In this way a link is established between reflexion and the dynamics of the neural network. The number of neurons in this network must be very large, since only in this case is the limit transition justified permitting us to write a differential equation. Regardless of the neural parallel, it turns out that the composition of functions describes not only multiple reflexion, but also a chain of abstract heat machines, which is in essence a particular "paraphrase" of the two first laws of thermodynamics. The model's logic suggests that the machines are analogues for consistent images of the self, and that the work they perform is an analogue for subjective experience.

In this way, the model of reflexion turns out to be indissolubly connected with such general concepts of complex systems as the formal chain of neurons and thermodynamics. This circumstance inclines us to the thought that the model's mathematical structure is that invariant which is inherent in all perfect systems, possessing, like the human being, a subjective world and the ability to multiply reflect it. If we accept this idea, it is natural to suppose that in creating its external self-description, the system uses the language of that invariant. In this case mathematical correspondences may constitute one of the aspects of this self-description, connected with the chain of heat machines. The work performed by these machines, in accord with the formal model, forms "double" geometrical progressions. This is a sequence of numbers consisting of two geometrical progressions with the same denominator. One progression corresponds to the odd members of the initial sequence, the other with the even ones. An example of this double geometrical progression is the following sequence with the denominator 2 :

\section{$2,3,4,6,8,12,16,24$}

Such sequences permit us to retrieve accurately the subjective state of a system. In this way, the model brings us to a concrete result. It predicts that in the signals created by perfect systems and bearing their self-description there may be a double geometrical progression.

\section{Rapid Burster}

We decided to try finding such sequences through analysis of the literature on the best known sources of alternating x-ray radiation. The broad class of such sources comprises bursters. Each of these objects, within the framework of our current knowledge, consists of two stars, one ordinary and one neutron, the latter drawing matter out of its companion. This accumulates on its surface, leading to periodic thermonuclear explosions which emit x-ray flashes. The idea that $\mathrm{x}$ - ray radiation can be used by intelligent beings for transmitting signals is not new: the American astronomer R. Corbet, in 1973, wrote that the radiation of bursters could be modulated, for example, by a metallic screen. These objects are few in number, and we can observe them throughout our galaxy.

In 1976 the strangest of these objects was discovered in the globular cluster Liller 1, and was named the Rapid Burster. Aside from the usual thermonuclear 
flashes, it also produces flashes of a second kind, whose physical mechanism has remained a mystery; there exists a point of view that they are connected with the appearance of certain instabilities in the accretion disk. One of the astonishing features of these flashes of the second type is the lack of correlation between their form, on the one hand, and their length and intensity, on the other. Such phenomena are very seldom encountered in non-living systems, though they may be common in artificial communication systems. For example, the same song may be performed loudly or quietly, quickly or slowly.

We discovered, that in 1985, the Japanese astronomer I. Tawara and his colleagues, in the process of analyzing the Rapid Burster's strange flashes, found in them precisely a double geometrical progression! Such sequences are formed not only by the height of peaks on the "slope" of their profiles, but also the time intervals between them. It was recently determined, that "regular" thermonuclear flashes are correlated with a radio source, found a distance of two and a half parsecs from the Rapid Burster (it is not out of the question, to be sure, that the disparity of x-ray and radio sources may be due to errors in the x-ray coordinates). Even before this it had been discovered that the radio source was located almost in the center of the spherical cluster. Does this all not indicate that the x-ray alternation of Rapid Burster is governed by intelligent beings living somewhere in that cluster? Alternatively, we may be observing the natural conscious activity of some intelligent subject of a non-biological character, those invariant characteristics spoken of earlier? (V.A. Lefebvre and Iu.N. Efremov, Astron. Astroph. Trans. V. 18, 335-342, 1999).

\section{The Inner Worlds of Black Holes}

The efforts of scientists working to unify the theory of relativity with quantum mechanics are currently focused on models of black holes - extremely dense objects, capable of "crushing" the space around them in such a way that they cannot release either matter, or radiation, or information. Nearly all researchers are convinced, that black holes exist in the cores of many galaxies, including our own. The mass of such black holes are millions of times that of the Sun. Nevertheless, each galaxy must also contain many black holes whose mass is on the order of ten times that of the Sun - these are produced by massive stars as a result of gravitational collapse following the exhaustion of their nuclear energy resources. Such stars usually rotate quickly around their axes.

A theoretical model of the collapse of a rotating body was constructed in 1963 by R. Kerr. It provides a description of the spatio-temporal properties found above the horizon of a black hole. The purely mathematical model, however, can be analytically continued to an area beyond this horizon. This area is fundamentally inaccessible to observation by an outside observer. For this reason, it does not have the status of reality, from a purely instrumental point of view. If we jump ahead, we will note that this exceptional character of black holes is, at least in one aspect, similar to the subjective world of the human being. This world, for the external observer, that is, for another human 
being, also has no operationally definable status as existent. We still do not know whether there is an experiment which would enable us to distinguish a being, possessed of subjectivity, from a soulless but highly refined automaton.

The interpretation of Kerr's model of black holes has shown that this area possesses, for internal observers, a number of remarkable features. The inside of a black holes is a complex system of infinite universes containing their own black holes. Within this system it is possible to distinguish a set of black holes sequentially inserted one within the other. Inside each of these black holes there is a pair of universes, such that distances within one of them are measured in positive numbers, and in negative numbers in the other. The components of this paradoxical pair (from a common-sense viewpoint) can be called the "positive universe" and "negative universe". These universes are separated by a singularity - a region of the world in which the density of matter and the curvature of space are equal to infinity (see J. Gribbin, Unveiling the Edge of Time, Three Rivers Press, New York, 1992).

The great surprise for physicists working in the field of quantum gravity was the theoretical conclusion that black holes must evaporate (Ia.B. Zeldovich, A. Starobinsky, S. Hawking). This conclusion followed on the discovery of connections between black holes and, simultaneously, both thermodynamics, and quantum mechanics. The second surprise arose, when S. Hawking suggested the possibility that the absorption of matter and radiation by black holes might lead to the disappearance of some quantity of information from our world. As we know, the most general description of a physical system is given by the equations of quantum mechanics. These equations are reversible, when they relate to micro processes. They allow us, with equal accuracy, to predict the future states of a quantum system and to retrieve past ones. It turns out, however, that if there is a black hole in a physical system, the equations describing micro processes, cannot simultaneously describe both the past and the future of the physical system. This means, from a formal viewpoint, that there occurs a disappearance of part of the information.

Further theoretical inquiries, conducted by the Dutch physicist J. t' Hooft, have nevertheless shown that such a loss is incompatible with the law of conservation of energy. This contradiction, which has been dubbed the "information paradox," threatened to destroy quantum mechanics, which is the basis for our modern scientific view of the world (L. Susskind, Black Holes and the Information Paradox; Scientific American, April 1997). It is therefore no surprise that serious steps were undertaken to resolve the information paradox. At first it was proposed that the information absorbed by the black hole might be returned in its process of evaporation. In this case, however, the emission of particles from various parts of space surrounding the horizon of the black hole would have to be correlated, and it remains wholly uncertain how such a correlation might be achieved. A new way of resolving the information paradox was suggested not long ago by the American physicist L. Susskind, who constructed a holographic model of a black hole.

Within this model, information does not disappear in the depths of a black hole. It is "deposited" on its shell or horizon. In other words, the quantity of 
information on the shell is always precisely equal to the quantity of information in the substance passing over the horizon. In this way, the shell of the hole is a particular "text," fixing the complexity of the flow of substance passing over the horizon. We see that black holes possess certain characteristics of an information system.

Another step toward the understanding of black holes was Susskind's and t' Hooft's generalization of Nils Bohr's principle of complementarity. The shell of a black hole, together with the information recorded in it, are a reality for the external observer. An observer, however, falling freely toward the center of the black hole, discovers no layer of information at the moment of crossing its threshold. This object, for him, does not have the status of a real existent. Nevertheless such a distinction of realities for two observers does not present logical difficulties, since there is no contact possible between them. The observers have no way to counterpose the results of their individual observations. The fact, that there is no information shell for the internal observer, does not signify by any means that we cannot regard it as a material object. We can ascribe the status of an existent to it only because it is real for the external observer, in exactly the same way as we agree to consider the electron a particle, despite the fact that in many experiments it behaves like a wave.

Let us recall here that, in accord with Kerr's model, inside the black hole there is found a sequence of black holes inserted one within the other. Let us now place an observer inside each of these holes, each capable of observing the subsequent black hole from without. Naturally, the principle of complementarity is generalized to include such observers. As a result, we arrive at a series of viewpoints complementarity with respect to one another, connected with the initial black hole.

\section{Black Holes and the Model of a Reflexive Sub- ject}

Returning from black holes to modern psychology, we can see a striking parallel between the inner world of a black hole in Kerr's model and the psychological model of a multiply self-aware subject. In both models there is a sequence of elements one within another, connected with the positions of observers related by complementarity. Each element contains an unsymmetrical pair (positive and negative universes in the black hole, positive and negative states in the model of the subject). In both cases, from the viewpoint of an external observer there exists a thermodynamic description of the system. Moreover, there are reasons to think that holographic models might turn out to be effective both for studying the brain and for investigating black holes.

Future research should provide an answer to the question of whether there exist deeper parallels between the model of black holes and the model of the subject. The establishment of such parallels might be a substantive step towards a fundamental unification: the inclusion of the intelligent subject in our physical 
worldview. Such parallels, however, have not yet been found. For this reason, our further deliberations on this theme are purely speculative.

\section{A Not-Quite-Scientific Hypothesis}

Nonetheless, we will indulge ourselves in the consideration of a fictive scenario. Since black holes have "emptiness" for the internal world, they can be turned, by a civilization like that on Earth, into a gigantic individual, a super-personality, capable of multiple self-awareness and preserving on its horizon (which may be an analogue of the brain) all the information accumulated by the civilization.

Let us emphasize that we are speaking of a black hole as of the physical basis of a single gigantic personality but not as of a new house into which individuals are moving to set up a civilization. The inner space of a black hole is the subjective world of this individual, which has no reality for an external observer. Everything found in this subjective world constitutes the "imagination" of the giant.

Such a "subject," being the successor to biological civilization, may be capable of self- development and, more importantly, of self-reproduction; in other words, black holes may give rise to other black holes. Quantum evaporation finally destroys the "intelligent" subject, whose body is the black hole. Nevertheless, it may exist for an unimaginably long time, even by cosmic standards. This, in fact, may be the reason that civilizations seek to transform themselves into such "almost eternal" objects. Yet how the black holes might give birth to a new generation?

According to modern views, for the appearance of a black hole it is essential that the mass of the collapsing star be at least three or four times that of the Sun. The lifespan of massive stars is very short on a cosmic scale, only a few million years. In this time a large portion of the nuclear fuel in the depths of such a star is exhausted. For this reason the light pressure weakens, the upper levels of the star collapse inward, and there is a huge explosion, called the flash of a supernova. The star's nucleus is crushed and turned into a black hole.

The actual physical mechanism for the appearance of massive stars is still not wholly understood. We know only that stars appear as the result of a condensation of interstellar gas. Apparently, in order for this condensation to produce a massive star, an external force is needed to raise the pressure in the cloud of gas. The formation region of massive stars is, as a rule, concentrated in the spiral arms of the galaxy; moreover, in the lifespan of a single galaxy there occur the births and deaths of many generations of massive stars. The

spiral pattern of symmetrical and long arms is a wave of increased density of stars and gases, which rotates like a solid body, with a constant angle speed, and on meeting the clouds of gas of the giant disk with already accumulated in the arms and there is a rise in pressure in the gas, leading ultimately to the formation of massive stars.

Is it possible to suppose that one of the tasks facing a cosmic subject would be to organize the "production" of massive stars, which sufficiently quickly, 
over millions of years, turn into black holes by natural means? Of course, the process of this creation also must be entirely natural - there must be some way to initiate the rise in density of the gas clouds. A powerful explosion in the gas medium is clearly the most economical way to initiate the formation of stars. Such explosions can be observed, and black holes may be linked in the most direct way with their appearance. Moreover, we can even speculate that many massive stars - future black holes - are born as a result of the death of a given black hole (following its merging with another component of the binary system of compact objects) and the appearance of a new black hole. It is believed that super-powerful explosions occur at the same time, accompanied by flashes of gamma-radiation.

\section{$9 \quad$ Star Arcs and Galactic Rings}

Not only a spiral wave of density, but also the flash of a supernova star lead to the appearance of a spherical wave of raised density gas, in which subsequently young stars take form. This mechanism is active in irregular galaxies. It has been several decades since the closest of them - the Large Magellanic Cloud (LMC) revealed strange structures called giant star arcs. They have been recognized since then in a number of other galaxies. These are huge arc-shaped young star complexes, with a curvature radius of 200-300 ps. In the centers of these immense star arcs there are no star clusters containing enough supernovae or 0-stars in them necessary to form arc- shaped star complexes. The suggestion has been put forward that these arcs are the product of a superpowerful explosion, creating a spherical wave of density in the cloud of gas. This hypothesis has been supported by the discovery of gamma-bursts, since they testify that there may occur even more powerful explosions in the Universe than those of supernovae.

Nevertheless there is one fact, showing that such a simple version of the origin of star arcs, at least, is incomplete. In the LMC and two other cases the arcs are formed by groups of two to four arcs. The super-powerful explosions are a fairly rare phenomenon even in cosmic terms. One might ask, what causes their appearance so close to one another, in only one part of the galaxy and with an interval of only about 10 million years? One of the authors (Iu.N. Efremov, Letters to the Astron. J. V. 25, 100-107, 1999; HERALD of Russian Acad. of Sci. Vestnik RAN, V.70, 314-323, 2000) put forward the hypothesis, that the progenitors of these objects, producing immense explosions, are born in a dense cluster and ejected from them before they explode. These are the characteristics to be expected in a binary system of compact objects - neutron stars or black holes, whose merging following the transfer of energy from orbital movement into gravitational wave produces just such gamma-bursts. Numerous massive stars in star arcs born from superexplosions turn ultimately into black holes.

Yet is the concentration of star arcs produced by superexplosions in close proximity to one another not the result of the coordinated activity of groups of intelligent black holes? If we accept this hypothesis, then it may be expected 
that there exist entire galaxies with the clear signs of the collective coordination of processes of production of new massive stars, from which black holes appear as if hatched from the eggs. For some reason their "natural" appearance is not enough, or, on the other hand, they need to show up precisely in that location, so that measures are taken to stimulate a local production of massive stars.

We know of an entire galaxy, with features inviting such an explanation the Cartwheel. It consists of a nucleus and two concentric rings. The nucleus and inner ring are formed out of old stars with little mass, whereas the outer ring consists of bright, young massive stars. From the internal ring to the outer there are "spokes" (giving the galaxy its name), at the same time these spokes are directed along tangents to the inner ring.

This galaxy has long attracted the attention of scientists. Pictures taken with the Hubble Telescope, have permitted a more detailed inspection of fine details in the inner ring. It turns out that there are objects there resembling comets in form. These objects seem to have a head and a tail, with the latter in many cases seeming to pass gradually into a spoke. The proposal was put forward that "comets" these are the traces of a substance falling toward the center of the galaxy. Nevertheless, such a hypothesis cannot explain the cometlike form of these traces. The following suspicion arises: are we not observing the remnants of synchronous directed explosions, whose purpose is to create a ring-shaped wave of density, leading to the formation of a large number of young massive stars in the outer ring, from which subsequently "ripen" black holes? If that is so, then, looking at the Cartwheel, we are observing a wave of intelligence, which was written about a quarter-century ago by Hart and Shklovsky. Yet the possessors of this intelligence are not biological organisms, but black holes. And the birth of new black holes is marked by fireworks of gamma-bursts, the most powerful explosions known in all the Universe.

\section{The Presumption of Naturality}

There are, of course, also natural explanations for groups of star arcs and the strange wheel- shaped galaxy. Near the group of arcs in the LMC there is in fact an old and massive star cluster, from which compact binary systems with a neutron star or black hole may have escaped, whose fusion led to the superpowerful explosion and gamma-ray burst. The unusual "Wheel" structure could have arisen due to the passage through this galaxy's center of another, smaller galaxy, creating a shock wave, and thus an active process of star formation, appearing to us as the outer ring.

One way or another, both the star arcs and galaxy with a ring around it are a highly strange formation, and they may be connected with the activity of a cosmic intelligence, perhaps by some means as yet completely unfathomable to us... Both "natural" hypotheses raise difficulties. Not far from the second group of giant star arcs, in the spiral galaxy NGC 6946, there are, it would seem, no suitable clusters, as will soon be verified by the Hubble Space Telescope. This galaxy possesses a few other peculiarities as well - extremely bright or adjacent 
remnants of supernovae, the spiral arms of an intensified magnetic field, not coinciding with the arms, optically visible...

We get the impression that there are galaxies in which the percentage of unusual objects - star arcs, rich young clusters or unusual supernovae and their remnants - is significantly higher than in others. The intensity of formation of massive stars in them is always elevated, and such objects are usually connected precisely with a high rate of star formation. This might be explained by high gas content, but these galaxies do not have a higher relative mass of gas than many others having no such peculiarities. Does not then NGC 6946 and Cartwheel belong to the number of galaxies, gripped by the Great Ring of intelligence...

The "presumption of naturality" spoken of by I.S. Shklovsky, the sorely needed attempts to find "natural" explanations of things and phenomena should not be abolutized or turned into a prohibition on flights of fantasy. With this thought, expressed in abstract form, everyone is eager to agree, yet as soon as we refer to a specific object provoking suspicion, the fantasizer begins to attract strange looks and risks the loss of his scientific reputation... Yet there is no other way than to keep searching and investigating all strange objects - always keeping in mind the possibility that we will encounter the traces of activity of intelligent subjects (see, for example, N.S. Kardashev, Voprosy filosofii, 12, 1977).

Let us remember that even artificially created objects and phenomena are unquestionably subject to the laws of physics, which are definitely known to us in areas remote from singularities. Many believe that we have long been the witnesses of activity of another intelligence, but do not realize it. Thus suspicion has fallen on the active cores of galaxies, possessing, like Cyg A, long narrow jets (sometimes up to several megaparsecs in length), from which are emitted a substance with subrelative speeds. The collimation mechanism of emissions from quasars and radiogalaxies, from object SS433 and young stars, - and possibly, also from objects generating gamma-bursts - the mechanism that makes these jets so narrow is not yet completely clear. The phenomenon is obviously natural, since it is encountered so frequently in nature - writes the American astrophysicist B. Paczynsky (Preprint astro-phys/9909048, 4 Nov 1999). Yet do these words not signify that bizarre thoughts occur not only to hopeless crackpots... 